\title{
Laguerre Gaussian filters in Reverse Time Migration image reconstruction
}

\author{
Juan Guillermo Paniagua (*), Universidad EAFIT
}

Daniel Sierra-Sosa, Universidad EAFIT

Copyright 2016, SBGf - Sociedade Brasileira de Geofísica

Este texto foi preparado para a apresentação no VII Simpósio Brasileiro de Geofísica, Ouro Preto, 25 a 27 de outubro de 2016. Seu conteúdo foi revisado pelo Comitê Técnico do VII SimBGf, mas não necessariamente representa a opinião da SBGf ou de seus associados. É proibida a reprodução total ou parcial deste material para propósitos comerciais sem prévia autorização da SBGf.

\begin{abstract}
Reverse time migration (RTM) solves the acoustic or elastic wave equation by means of the extrapolation from source and receiver wavefield in time. A migrated image is obtained by applying some criteria known as imaging condition. The zero lag cross-correlation between source and receiver wavefields is the commonly used imaging condition. However, this imaging condition produces lowspatial-frequency noise or artifacts, due to the strong contrasts in velocity field (Pestana et al., 2014). Several imaging techniques have been proposed to reduce the artifacts occurrence. Derivative operators as Laplacian are the most frequently used. In this work, we propose the usage of a technique based on a spiral phase filter ranging from 0 to $2 \pi$, and a toroidal amplitude bandpass filter, known as Laguerre-Gauss transform. Through numerical experiments we present the application of this particular filter on SEG EAGE salt model and Sigsbee 2A model. We also present evidences that this method improves RTM images by reducing the artifacts and notably enhance the reflective events.
\end{abstract}

\section{Introduction}

Reverse time migration solves the two-way acoustic or elastic wave equation, by the propagation in time domain of the source wavefield in forward direction, and of the receiver wavefield in backward direction. The migrated image is obtained by the cross-correlation between source and receiver wavefields summed over the sources (Claerbout, 1985).

The cross-correlation imaging condition produces lowfrequency noise called artifacts due to the superposition between waves (such as head waves, diving waves and backscattered waves) immersed in the source and receiver wavefields and the migrated images amplitude.

To reduce the artifacts, several techniques have been proposed. Youn and Zhou (2001) used the Laplacian image reconstruction to process each frame from correlation for an individual shot recorded, Fletcher et al. (2005), added a directional damping term to the nonreflecting wave equation proposed by Baysal et al. (1984) and Yoon and Marfurt (2006) used the Poynting vectors to improve the cross-correlation imaging condition.
Kaelin and Guitton (2006) normalized the image of the cross-correlation diving by the source or the receiver illumination, Guitton et al. (2007) used the smooth imaging condition and the least square attenuation method, Costa et al. (2009) combined the obliquity factor weight and illumination compensation in the imaging condition, Whitmore and Crawley (2012) used the inverse scattering theory to attenuate the backscattered waves and Pestana et al. (2014) based on the relation of inversion and imaging, proposed the impedance sensitivity kernel imaging condition combined with Poynting vector.

In this paper we propose a method to improve the migrated image and diminish the artifacts occurrence by applying a Laguerre Gauss filter with a spiral phase filter to implement a Radial Hilbert transform to process the cross-correlation images. First, we describe the Cross correlation imaging condition and the Laplacian filtering, which is a simple and popular way to remove the artifacts in RTM images. Second, the proposed method is described. Finally, we compare the images obtained by cross correlation imaging condition, laplacian filtering, and the Laguerre Gauss filtering applied to two synthetic datasets, to present evidences from the effectiveness of our imaging implementation to reduce the low-frequency spatial noise.

\section{Cross-correlation imaging condition}

The zero-lag cross-correlation between the extrapoled source and receiver wavefields is the imaging condition conventionally used in RTM. The cross-correlation imaging condition was proposed originally by Claerbout (1971, 1985) and as defined as follows:

$$
I_{c c}(x, z)=\sum_{j=1}^{s_{\max }} \sum_{i=1}^{t_{\max }} S\left(x, z ; t_{i} ; s_{j}\right) R\left(x, z ; t_{i} ; s_{j}\right)
$$

where $z$ and $x$ denote depth and horizontal axis respectively, $t$ is time, $S\left(x, z ; t_{i} ; s_{j}\right)$ is the forward propagated source wavefield, $R\left(x, z ; t_{i} ; s_{j}\right)$ is the backward propagated receiver wavefield, $t_{\max }$ is the total time, $s_{\max }$ is the total number of sources, and $I_{c c}$ is the crosscorrelation image.

Several implementations of RTM using the imaging condition of (1) have been reported by Baysal et al. (1983, 1984), Youn and Zhou (2001), Kaelin and Guitton (2006), among others.

This method can be affected by the backscattered and turning waves in the modeling process, which cause incident and reflected wavefields to be in phase at locations that not correspond to actual reflection points. These wavefields cause strong correlation noise in the 
seismic image (Artifacts) (Whitmore and Crawley, 2012). These artifacts occur most frequently in shallow parts and hard interfaces in the velocity model, and can mask important details in the image.

\section{Laplacian filtering}

Second-order derivative edge detection techniques are based on spatial second-order differentiation to enhance edges. An edge is marked if a significant spatial change occurs in the second derivative (Pratt, 2001). The edge Laplacian (Laplacian image reconstruction) of an image function $F(x, y)$ is defined as:

$$
G(x, y)=-\nabla^{2}\{F(x, y)\}
$$

where, the Laplacian operator is:

$$
\nabla^{2}=\frac{\partial^{2}}{\partial x^{2}}+\frac{\partial^{2}}{\partial y^{2}}
$$

$G(x, y)$ is zero if $F(x, y)$ is constant or the amplitude changes linearly. If the change rate of $F(x, y)$ is greater than zero $G(x, y)$ exhibits a sign change at the point of inflection in $F(x, y)$. The zero crossing of $G(x, y)$ indicates the presence of an edge. The negative sign from (2) indicates that the zero crossing of $G(x, y)$ has a positive slope for an edge whose amplitude increases from left to right or bottom to top in an image (Pratt, 2001). Youn and Zhou (2001) used the Laplacian image reconstruction operator to correlated image frames. The Laplacian image reconstruction is given by:

$$
I_{L P}(x, z)=\frac{\partial^{2}}{\partial x^{2}} I_{c c}(x, z)+\frac{\partial^{2}}{\partial z^{2}} I_{c c}(x, z)
$$

where $I_{c c}(x, z)$ is the image frame registered (Cross correlation image). An effect associated with the application of the Laplacian operator is a $90^{\circ}$ phase shift and an amplitude change due to the second-order differential.

\section{Laguerre-Gauss filtering}

Linear integral transforms have been used in multiple knowledge fields such as quantum mechanics, quantum theory fields, quantum dispersion, viscoelasticity, circuit theory, dielectric theory, magnetic resonance, optical metrology, among others (Macdonald and Brachman, 1956, Sierra-Sosa et. Al, 2013, 2014, Angel-Toro, 2013). In general, these transforms turn a given function $f(z)$ where the $z$ variable may be complex or real valued, into another function $g(\omega)$ where the variable $\mathrm{w}$ also may be complex or real valued, these transforms can be defined as:

$$
g(\omega)=\int_{C_{n}} K_{n}(\omega, z) f(z) d z
$$

where $K_{n}$ with $n=1,2$ are generic functions from the variables $z$ and $\omega$ known as transform kernel, for both the transform with $n=1$ as for its inverse with $n=2 ; C_{n}$ denotes the path in the complex plane. In particular, if $f(z)$ is well-known and $K_{1}$ and $C_{1}$ are specified, it is possible to obtain $g(\omega)$ if the function is integrable. On the other hand, this equation is also a linear integral transform for $f(z)$ when $g(\omega), K_{2}$ and $C_{2}$ are specified. For each integral transform there exist a relation that turns the transformed function into the original function, usually this relation is also an integral transform, that may be written in terms of algebraic operations. There exist a biunivocal relation among the function and its transform (Debnath and Bhatta, 2010).

If kernels $K_{1}$ and $K_{2}$ are equal and the integration paths between the transform and its inverse are equal, the relation between $f(z)$ and $g(\omega)$ are reciprocal and known as conjugated functions. When these functions are identical except for the sign, like in Hilbert transform, the relations are contra-reciprocal. The integral transforms are useful to obtain a complex distribution similar to the phase from real valued functions, these distributions are known as pseudo-phase maps (Freund and Freilikher, 1997). Allowing to obtain an analytic signal from a real valued function, analytic signal concept was introduced by Gabor in communications theory for 1-dimensional signals (Debnath and Bhatta, 2010). Usually, an analytic signal is defined by the difference of the real part of the original signal and its mean value, and an imaginary part obtained by using an integral transform from real signal.

Laguerre Gauss transform kernel uses a pure-phase function with a vortex structure in frequency domain, defined as $\beta(f x, f y)=\arctan (f y / f x)$. The particular property from this spiral phase function is that is composed by a heavy-side function with a $\pi$ gap when crossing the origin in every angular direction. In the amplitude the kernel includes a toroidal geometry doing a band-pass filtering depicted in Figure 1.

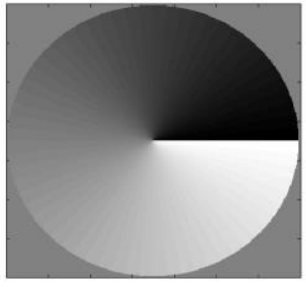

(a)

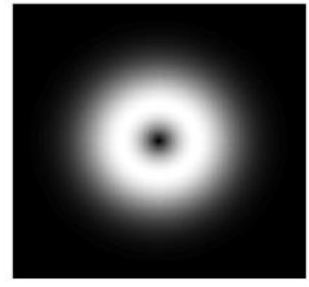

(b)
Figure 1. (a) Spiral Phase Function (b) Toroidal amplitude

Let $I(x, y)$ be the original intensity distribution from an image and $\hat{I}\left(f_{x}, f_{y}\right)$ its Fourier transform. $I(x, y)$ may be related with its respective analytic signal $\tilde{I}(x, y)$ by using a transformation kernel $L G\left(f_{x}, f_{y}\right)$

$$
\begin{gathered}
\tilde{I}(x, y)=\iint_{-\infty}^{\infty} L G\left(f_{x}, f_{y}\right) \hat{I}\left(f_{x}, f_{y}\right) e^{2 \pi i\left(f_{x} x, f_{y} y\right)} d f_{x} d f_{y} \\
L G\left(f_{x}, f_{y}\right)=\left(f_{x}+i f_{y}\right) e^{-\left(\frac{f_{x}^{2}+f_{y}^{2}}{\omega^{2}}\right)}=\rho e^{-\left(\frac{\rho^{2}}{\omega^{2}}\right)} e^{i \beta}
\end{gathered}
$$


where $\rho=\sqrt{f_{x}^{2}+f_{y}^{2}}$ and $\beta(f x, f y)=\arctan (f y / f x)$ are the polar coordinates in the spatial frequency domain. The bandpass filter can be controlled by choosing the bandwidth parameter $\omega$ (Wang et al., 2006), which changes the spatial frequency distribution in the obtained complex field. In this case, $\omega$ tends to one in order to preserve image spatial frequency distribution and perform the bandpass filter component from Laguerre Gauss.

The Laguerre-Gauss filter allows to realize anisotropic radial Hilbert transform without resolution loss (Gou et al., 2006), and excluding any DC component of the original input function.

\section{Numerical examples}

We show the results for two synthetic datasets: 2D SEGEAGE salt model and Sigsbee 2A model applying the Laguerre-Gauss filter introduced above. We applied RTM using own $\mathrm{C}$ parallelized algorithms implemented with a high order finite difference scheme (Eight order). The RTM images have been obtained using the zero lag crosscorrelation imaging condition, the Laplacian filtering and the Laguerre-Gauss filtering.

The 2D SEG-EAGE velocity model is shown in Figure 2.

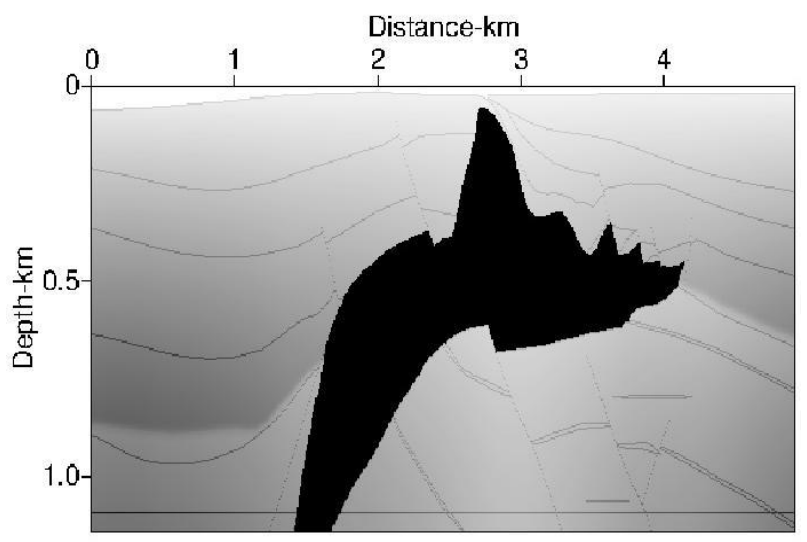

Figure 2. 2D SEG-EAGE velocity model

In Figure 3a the RTM image obtained from zero lag cross correlation imaging condition given by equation 1 is shown. The image was obtained using only ten shots.

The image is contaminated with artifacts in the shallow parts and near the salt body, and white spots on the bottom caused by backscattering hiding some details of subsurface structures.

In Figure $3 b$ we show the RTM result using the Laplacian filtering which shows a good reduction of artifacts. To apply this filter reduces low frequency information and increases the high frequency noise (Guitton et al., 2007).

Figure $3 c$ presents the image from using the LaguerreGauss filtering presented in equations 6 and 7 . We demonstrate that the Laguerre-Gauss filter removes the undesired low frequency noise in the RTM images.

Applying the Laguerre-Gauss Filter to cross correlation image the resulting image is improved: subsurface structures are more defined and the edges of the salt dome are enhanced. The artifacts in shallow parts and near the top salt dome are significantly reduced.

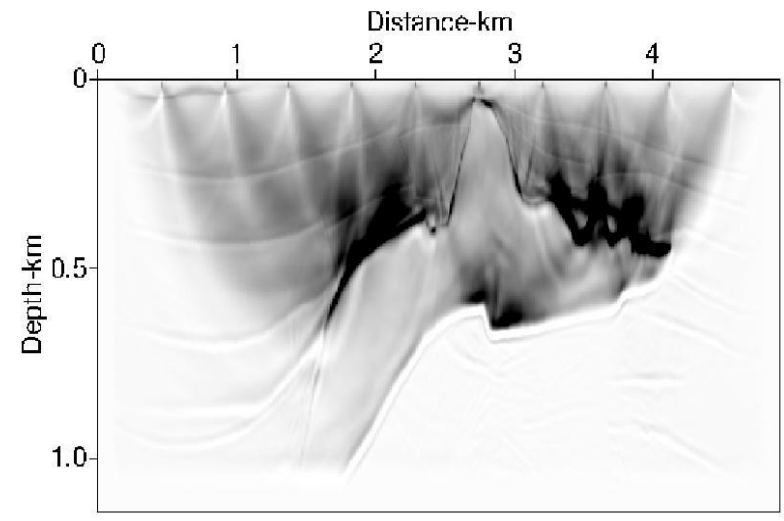

(a)

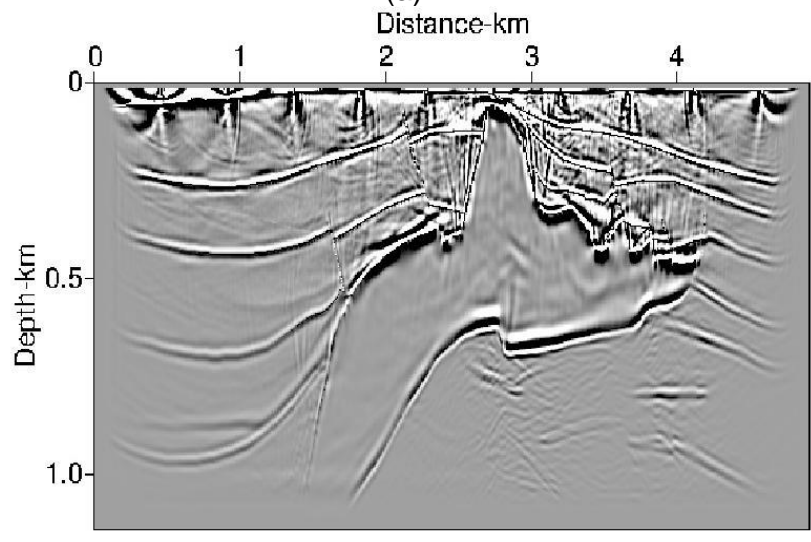

(b)

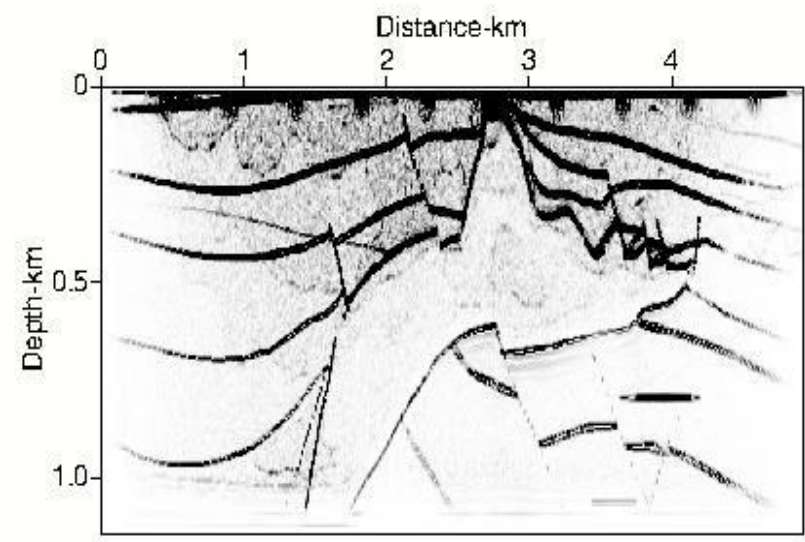

(c)

Figure 3. 2D SEG-EAGE RTM using: a) Cross correlation imaging condition b) Cross correlation imaging condition plus Laplacian filter c) Cross correlation imaging condition plus Laguerre-Gauss filter

In Figure 4, a close up view of two sections (Marked with yellow boxes) of the migrated image are presented. These were chosen due to its structure complexity. The artifacts reduction in images become apparent, by conducting the proposed technique images are improved and the salt body flanks are enhanced. 


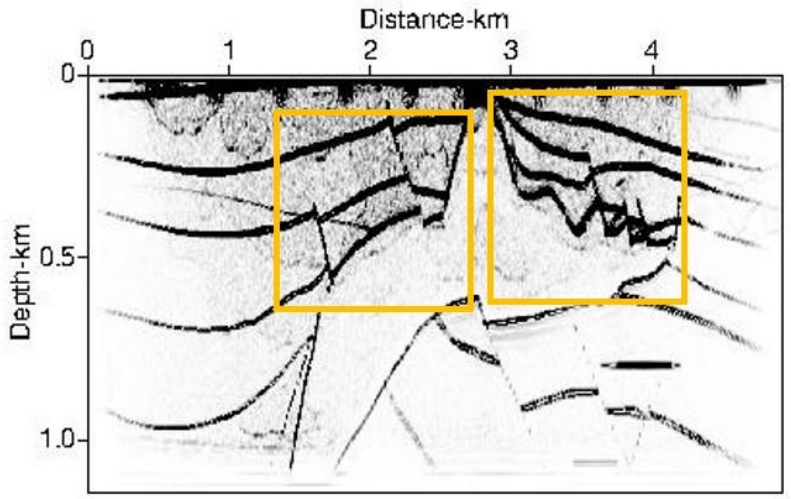

(a)

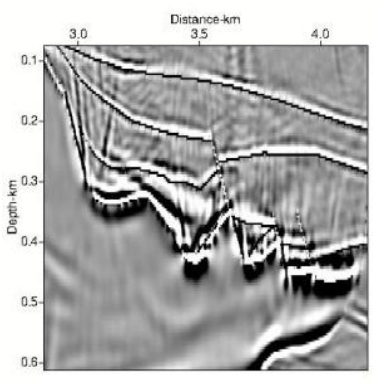

(b)

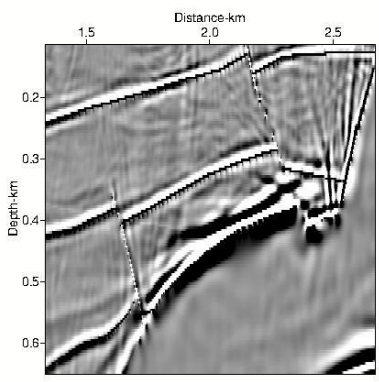

(d)

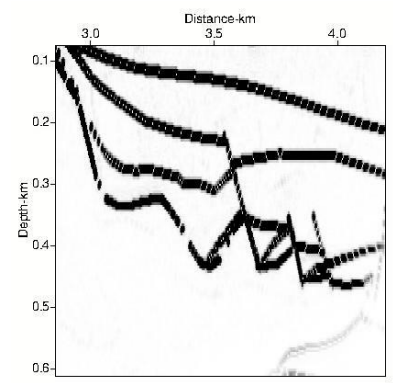

(c)

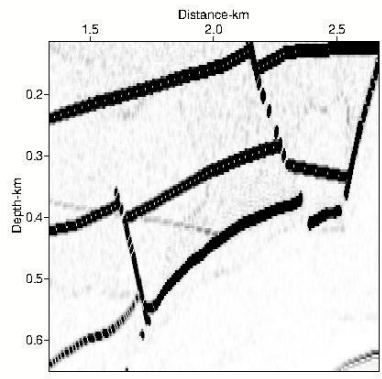

(e)
Figure 4. Detail of 2D SEG-EAGE RTM image with: a) Details of the outline regions; b) and d) Laplacian filtering; c) and e) Laguerre-Gauss filtering

Similarly, we apply the Laguerre Gauss filtering to 2D Sigsbee 2A model. Sigsbee $2 A$ is a synthetic model of deep water in the Gulf of Mexico. It is characterized by the complex salt shape with rugose salt top found in this area. The velocity model is shown in Figure 5 .

In Figure 6 is shown a comparison of images obtained by zero lag cross-correlation imaging condition, laplacian and La-guerre Gauss filtering. The RTM image was obtained using only sixteen shots distributed along the surface. Figure $6 \mathrm{a}$ shows the conventional cross correlation image where we can be observed that the low frequency noise is very strong near the salt body. Applying the Iaplacian filtering, the low frequency noise is reduced significantly. This effect is presented in Figure $6 \mathrm{~b}$. The result obtained by Laguerre-Gauss filtering is shown in Figure $6 \mathrm{c}$. The noise is strongly reduced near the salt body and the structures are enhanced. Likewise, the effect of sources in the surface are significantly attenuated.

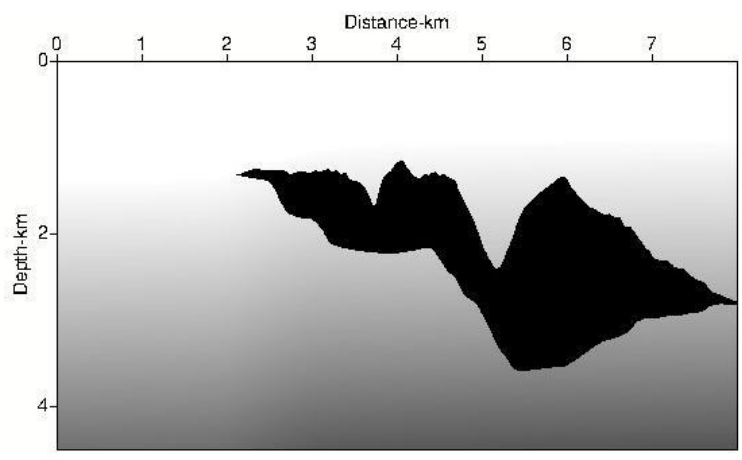

Figure 5. 2D Sigsbee $2 A$ velocity model

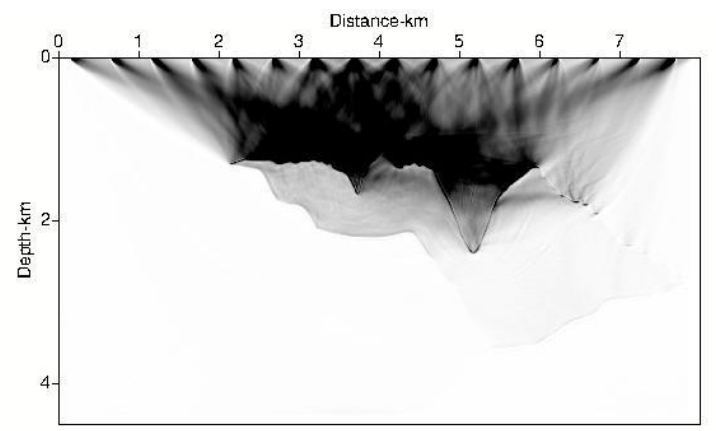

(a)

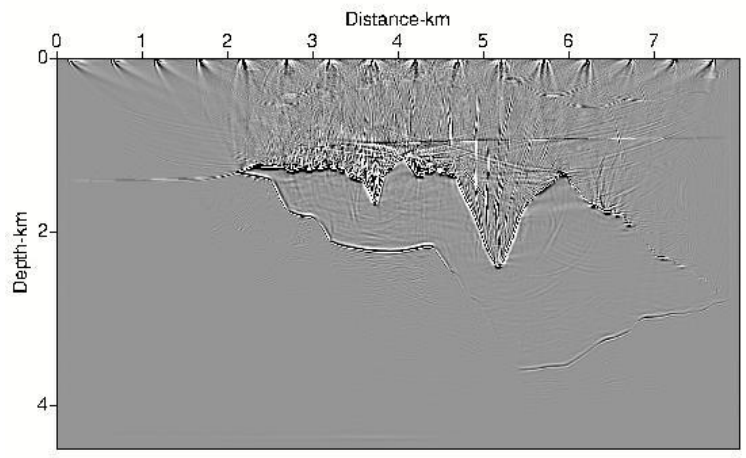

(b)

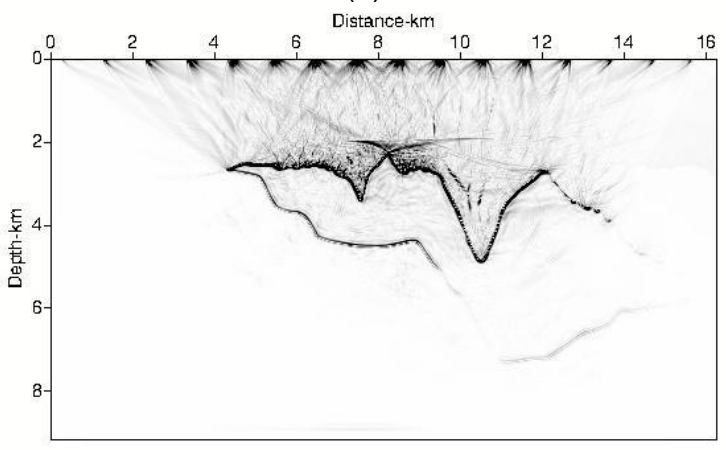

(c)

Figure 6. 2D Sigsbee 2A RTM using: a) Cross correlation imaging condition; b) Cross correlation imaging condition plus Laplacian filtering; c) Cross correlation imaging condition plus LaguerreGauss filtering. 
In order to verify that the results obtained by using the proposed technique match the velocity model interfaces, we proceed to overlap the found interface structures using red lines. Red lines are obtained eroding the contrast inverted Laguerre-Gauss grayscale images, by means of the convolution between the image and a disk with 2-pixel radius. The objects smaller than the mask disappear and a zero is assigned in its place, the positions of the remaining objects are assigned a one. To these binarized image a skeletonization process is applied by obtaining the distance transform of the image. The image skeleton lies along the transform singularities.

Figure 7 presents the interfaces obtained when using the proposed technique overlapped with the velocity model in a) for SEG-EAGE salt model and in b) for Sigsbee $2 A$ model, it should be noted the high correspondence between the obtained interfaces with those presented in each model.

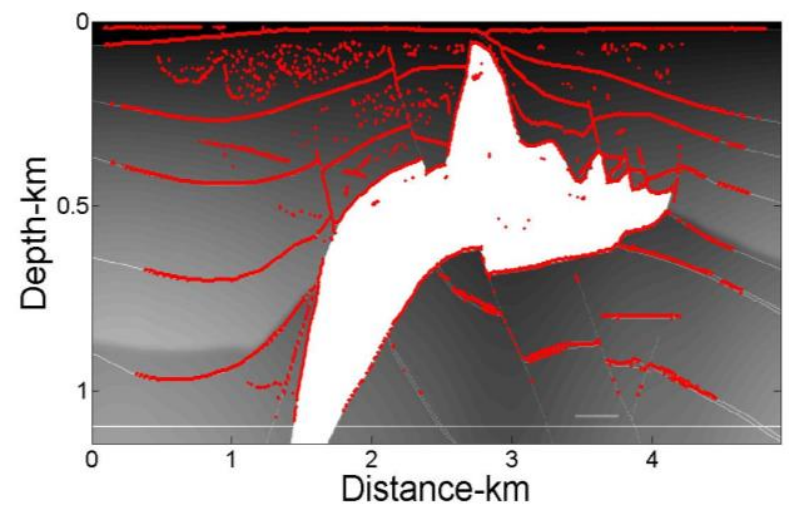

(a)

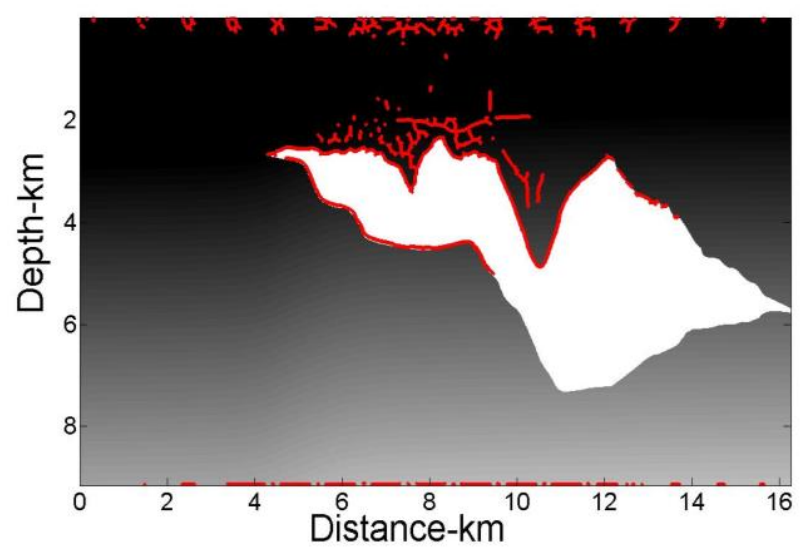

(b)

Figure 7. Comparison between images obtained with Laguerre Gauss filtering and the initial models: a) SEG-EAGE salt model; b) Sigsbee $2 A$ model.

\section{Conclusions}

We have proposed a technique to attenuate RTM artifacts after the zero-lag cross-correlation imaging condition. This method uses the Laguerre Gaussian filter to reduce the low-frequency spatial noise and enhances the edges in the image. To apply this filter to the image obtained with cross- correlation imaging condition, the low-frequency noise (artifacts) was removed and the reflective events were more defined. This filter enhances the edges without resolution loss.

\section{Acknowledgments}

We would like to express our gratitude with Colombian Oil Company ECOPETROL and COLCIENCIAS for supporting this work as a part of the research project grant No. 0266-2013.

\section{References}

Angel-Toro, L., Sierra-Sosa, D., Tebaldi, M., and Bolognini, N., 2013, In-plane displacement measurement in vortex metrology: JOSA A, 30, 3, 462-469-

Baysal, E., D. D. Kosloff, and J. W. Sherwood, 1984, A twoway nonreflecting wave equation: Geophysics, 49, 132141.

Baysal, E., D. D. Kosloff, and J. W. C. Sherwood, 1983, Reverse time migration: Geophysics, 48, 1514-1524.

Claerbout, J. F., 1971, Toward a unified theory of reflector mapping: $36,467-481$.

Claerbout, J. F., 1985, Imaging the Earth's interior: Blackwell Scientific Publications.

Costa, J., F. Silva, R. Alcántara, J. Schleicher, and A. Novais, 2009, Obliquity-correction imaging condition for reverse time migration: Geophysics, 74, S57-S66.

Debnath, L., and D. Bhatta, 2010, Integral transforms and their applications: CRC press.

Fletcher, R., P. Fowler, and P. Kitchenside, 2005, Suppressing artifacts in prestack reverse time migration: 75th International Annual Meeting, SEG, Expanded abstracts, 2049-2051.

Freund, I., and V. Freilikher, 1997, Parameterization of anisotropic vortices: Journal of the Optical Society of America A, 14, 1902-1910.

Gou, C., Y. Han, and J. Xu, 2006, Radial Hilbert transform with Laguerre-Gaussian spatial filters: Optics Letters, 31, 1394-1396.

Guitton, A., A. Valenciano, D. Bevc, and J. Claerbout, 2007, Smoothing imaging condition for shot-profile migration: Geophysics, 72, 149-154.

Kaelin, B., and A. Guitton, 2006, Imaging condition for reverse time migration: 76th International Annual Meeting and exposition, SEG, Expanded abstracts, 2594-2598.

Macdonald, J. R., and M. K. Brachman, 1956, Linear system integral transform relations: Reviews of modern physics, 28, 393-422.

Pestana, R., A. Dos Santos, and E. Araujo, 2014, RTM imaging condition using impedance sensitivity kernel combined with the Poynting vector: SEG Technical Program Expanded Abstracts, 3763-3768. 
Pratt, W. K., 2001, Digital image processing: Wiley Interscience.

Sierra-Sosa, D., Angel-Toro, L., Bolognini, N., and Tebaldi, M., 2013, Novel vortex-transform for high frequency modulated patterns: Optics Express, 21, 20, 23706-23711.

Sierra-Sosa, D., 2014, Estudio de singularidades de fase con aplicaciones a la metrología. Doctoral dissertation. Facultad de Ciencias Exactas Universidad Nacional de la Plata.

Wang, W., T. Yokozeki, R. Ishijima, M. Takeda, and S. G. Hanson, 2006, Optical vortex metrology based on the core structures of phase singularities in Laguerre-Gauss transform of a speckle pattern: Optics Express, 14, 1019510206.

Whitmore, N., and S. Crawley, 2012, Applications of RTM inverse scattering imaging conditions: 82nd Annual International Meeting, SEG, Expanded abstracts, 779-784.

Yoon, K., and K. Marfurt, 2006, Reverse time migration using the Poynting vector: Exploration Geophysics, 37, 102-107.

Youn, O., and H. Zhou, 2001, Depth imaging with multiples: Geophysics, 66, 246-255. 